\title{
CASO PRÁCTICO DE EVALUACIÓN DE IMPACTO AMBIENTAL DE CARRETERAS: VARIANTE DE BORRIOL (CASTELLÓN)
}

\author{
(PRACTICAL CASE ON ENVIRONMENTAL IMPACT ASSESSMENT OF ROADS: BYPASS OF \\ BORRIOL, CASTELLON)
}

María Luisa Domínguez González, Ingeniero de Caminos, Canales y Puertos

Ingeniería de Trazados y Estructuras, S.A. (INTRAESA)

ESPAÑA

Fecha de recepción: 6-X-95

$113-36$

\section{RESUMEN}

En las administraciones españolas, tanto en la central como en las autonómicas, el proceso de Evaluación de Impacto Ambiental comprende varias fases. En este articulo se describen todas ellas para el caso de una variante de carretera, la Variante de la C-238 a su paso por Borriol (Castellón), cuya titularidad corresponde a la Generalitat Valenciana. Se trata de uno de los procesos de Evaluación de Impacto más reciente de esta Administración Autonómica.

En primer lugar se exponen los puntos más destacados del Estudio de Impacto Ambiental incluido, como Documento Anejo, en el Proyecto Básico y de Expropiaciones "Autovía Castellón - La Pobla Tornesa (C-238). Tramo: Borriol Sur La Pobla Tornesa Norte", de fecha noviembre de 1993.

En el mes de julio de 1994 se sometió a Información Pública este Proyecto Básico. Con fecha 21 de octubre de 1994 la Direcció General de Qualitat Ambiental de la Consellería de Medi Ambient de la Generalitat Valenciana formuló la Declaración de Impacto Ambiental sobre el citado Proyecto Básico y de Expropiaciones. En este trabajo se comenta la casi total coincidencia de las medidas correctoras prescritas con las que se proponian en el Estudio de Impacto Ambiental.

En la actualidad, el Proyecto de Construcción "Variante de Borriol de la C-238 (Castellón)", ha sido aprobado por la autoridad competente y en fechas próximas se licitarán las obras objeto del mismo. Dichas obras incluyen las medidas correctoras de Impacto, que se exponen como conclusión de este trabajo.

\section{SUMMARY}

In all the spanish administrations, not only the central but also the authonomical ones, the Environmental Impact Assessment processe has some stages. In this article all this stages are described on the case of the new route of an existing road, the bypass of the C-238 road at Borriol, Castellon, whose owner is the Generalitat Valenciana. This is one of the most recent processes of Environmental Impact Assessment at this Authonomical Administration.

First of all, the most important aspects of the Environmental Impact Studie are shown; this Studie is included, as an Anexe Document, in the Basic Project "Highway between Castellon and La Pobla Tornesa (C-238). South Borriol - North La Pobla Tornesa", November 1993.

On July 1994, this Project passed the Public Information processe. On October the 21 $1^{\text {st }}$ 1994, the Direcció General de Qualitat Ambiental of the Conselleria de Medi Ambient of the Generalitat Valenciana edited the Environmental Impact Declaration about the Project. In this article, the almost total equality between the corrective measures it oblies to and the ones proposed in the Environmental Impact Studie are exposed.

Nowadays, the Constructive Project "Bypass of Borriol of the C-238 (Castellon)", has been approved by the authorities, and the works will start soon. This works include the impact corrective measures, which are explained as the conclusion of this work. 


\section{Introducción}

Desde que España entróa formar parte de las Comunidades Europeas, la normativa en cuestiones de Medio Ambiente se ha ido adaptando paulatinamente a la del resto de Europa. En el año 1985 fue aprobada por la CEE la Directiva 85/337/EEC, de 5 de julio, que establece la obligatoriedad de realización de este tipo de Estudios en el ámbito comunitario para determinados proyectos. El 30 de junio de 1986 se publicaba en el B.O.E.español el Real Decreto Legislativo 1302/1986, de 28 de junio, de evaluación de impacto ambiental, norma con rango de Ley, que desarrolla la Directiva. Posteriormente, se publica el R.D. $1131 / 88$, que desarrolla la anterior.

De acuerdo con esta normativa, para todos los Proyectos de "Construcción de autopistas, autovías, líneas de ferrocarril de largo recorrido, aeropuertos (.../...)", se deberá realizar un Estudio de Impacto Ambiental que acompañe al Estudio Informativo, en el caso de la Administración Central (M.O.P.T.M.A.) o, como en el caso de este artículo, al Proyecto Básico, figura equivalente en la Generalitat Valenciana. El proceso en esta Comunidad Autónoma es el siguiente:

\section{1. Redacción de un Proyecto Básico y de Expropiaciones}

Se encarga a una empresa consultora la elaboración de un ProyectoBásico y de Expropiaciones, en el cual se estudian y proponen una serie de alternativas de trazado a escala $1 / 5.000$.

Dicho Proyecto debe contener necesariamente un Estudio de Impacto Ambiental, en el que se analizan todas las alternativas propuestas permitiendo así su comparación desde el punto de vista medioambiental. Se califica cada una de las alternativas, y se recomienda una de ellas. Aquéllas cuyo impacto sea calificado como CRÍTICO deberán quedar descartadas del estudio global en el Proyecto Básico.

En el Proyecto Básico se lleva a cabo un análisis conjunto de todas las alternativas, donde las conclusiones del Estudio de Impacto Ambiental son un factor de peso, pero que deberá combinarse con otros factores, como el económico, social, político, etc.

En el Proyecto se desarrolla a una escala de mayor detalle, que en la mayoría de los casos es $1 / 1.000$, la solución recomendada, calculando un trazado en planta y alzado que cumpla con la Normativa vigente, y proponiendo la tipología más adecuada para las estructuras, obras de drenaje y todo tipo de elementos a proyectar.

Se hace un estudio en detalle de las expropiaciones que es preciso realizar para la construcción de esa alternativa, puesto que, caso de resultar aprobado el Proyecto Básico, se procede a expropiar a partir de los planos y las superficies de afección a cada propietario incluidas en el mismo.

El Estudio de Impacto Ambiental es uno de los Anejos a la Memoria del Proyecto Básico. Sin embargo se encuaderna en un tomo aparte para que pueda ser analizado por separado por los organismos y entidades que participarán en su aprobación

\subsection{Proceso de Información Pública. Formulación, si procede, de la Declaración de Impacto}

La Consellería d'Obres Publiques, Urbanisme i Transports (C.O.P.U.T.) somete la totalidad del Proyecto Básico a Información Pública; asimismo remite copias del Estudio de Impacto Ambiental a diversos organismos y entidades afectadas y con autoridad en la materia, como son Confederaciones Hidrográficas, Asociaciones Ecologistas, Universidades con Departamentos dedicados al tema, Asociaciones Culturales, Ayuntamientos,...

Esta fase tiene una duración de un mes, y durante la misma, aquellas personas que se encuentren en desacuerdo con cualquier propuesta o solución del Proyecto Básico tienen derecho a hacer todo tipo de alegaciones al mismo.

Al final de esta fase, la C.O.P.U.T. deberá responder a las alegaciones, con la colaboración de la Empresa consultora que elaboró el Proyecto. Se rectificarán o completarán aquellos aspectos del Proyecto que fuese preciso.

Con un margen de tiempo no inferior a 50 días a contar a partir del final del período de Información Pública, la Direcció General de Qualitat Ambiental de la Consellería de Medi Ambient formulará la Declaración de Impacto correspondiente. Deberá detallar las medidas correctoras de impacto que habrán de proyectarse, medirse y valorarse en el Proyecto de Construcción.

\subsection{Proyecto de Construcción}

Se encarga a una Empresa Consultora la redacción del Proyecto de Construcción en cuestión. Generalmente un mismo Proyecto Básico suele dar lugar a varios Proyectos de Construcción, que se refieren a tramos más cortos

Recoge todos los Documentos y cálculos necesarios para la total y correcta definición de las obras. Deberá incluir las medidas correctoras de impacto preceptuadas en la Declaración de Impacto, representadas en planos, especificadas en el Pliego de Prescripciones Técnicas Particulares, y medidas y valoradas como unidades de obra integrantes del Proyecto al igual que las específicas de construcción de la carretera.

Con esto termina el proceso de Evaluación de Impacto Ambiental de una carretera en la fase de Proyecto. 
A continuación se analizan estas fases sobre el caso concreto de la carretera $\mathrm{C}-238$ a su paso por la localidad de Borriol (Castellón).

\section{Proyecto básico. Estudio de impacto ambiental}

La carretera C-238, de titularidad autonómica, discurre por la provincia de Castellón, entre Castellón de la Plana y la carretera N-232, a unos $5 \mathrm{~km}$ de Sant Mateu. Borriol es la primera localidad, a $8 \mathrm{~km}$ de Castellón, por la que pasa la carretera en travesía, registrando un alto nivel de tráfico.

En el I Plan de Carreteras de la Comunidad Valenciana, 1986-1995, se sientan las bases de la red básica comunitaria, en la que se encuentra incluida la variante de Borriol. En el II Plan de Carreteras se determina que se proyectará como autovía, al formar parte del eje Castellón-La Pobla Tornesa-Albocasser-Ares-Morella, y del eje vertebrador de las Comarcas interiores del norte de la Comunidad Valenciana, eje Almenara-La Jana.

El Proyecto Básico y de Expropiaciones “Autovía CastellónLa Pobla Tornesa (C-238). Tramo: Borriol Sur-La Pobla Tornesa Norte" comprende el tramo de la carretera C-238 entre los P.K. $8+000$ y $20+000$, y consta de la variante de Borriol en autovía y la duplicación de la calzada existente desde el final de dicha variante hasta el final de la variante de La Pobla Tornesa. Este Proyecto fue redactado por la Empresa Ingeniería de Trazados y Estructuras, S.A. (INTRAESA) en noviembre de 1993.

Se considera dividido en tres tramos: la variante de Borriol, la duplicación de calzada del tramo Borriol-curvas de La Pobla Tornesa, y la conversión en autovía del tramo curvas y variante de La Pobla Tornesa.

Este artículo se centra en el primer tramo, puesto que aunque la Declaración de Impacto hace referencia a los tres, todavía no se ha redactado el Proyecto de Construcción de los dos últimos y, por tanto, el análisis del proceso completo se realiza para la variante de Borriol, de unos $3 \mathrm{~km}$ de longitud.

Se estudiaron cuatro alternativas de trazado, todas ellas al sur del casco urbano de Borriol, por considerarse impracticable cualquier trazado al norte debido a la existencia de unos cerros junto a la localidad. La toma de datos y elaboración del Inventario, por tanto, se hizo teniendo en cuenta los cuatro corredores. El ámbito geográfico es único, pues todas las alternativas están incluidas en la Plana Alta de Castellón. Se recopiló información sobre estudios anteriores, así como el Planeamiento previsto en la revisión de las Normas Subsidiarias de Borriol.

El inventario de factores ambientales que podían verse afectados con el Proyecto pasaba revista a:

\section{Medio Físico:}

Medio inerte: atmósfera-clima, tierra, agua, paisaje. Medio biótico: vegetación, fauna, flora, suelo productivo.

\section{Medio Socioeconómico:}

Población, Economía, Sistema de núcleos habitados, patrimonio histórico-artístico, infraestructura.

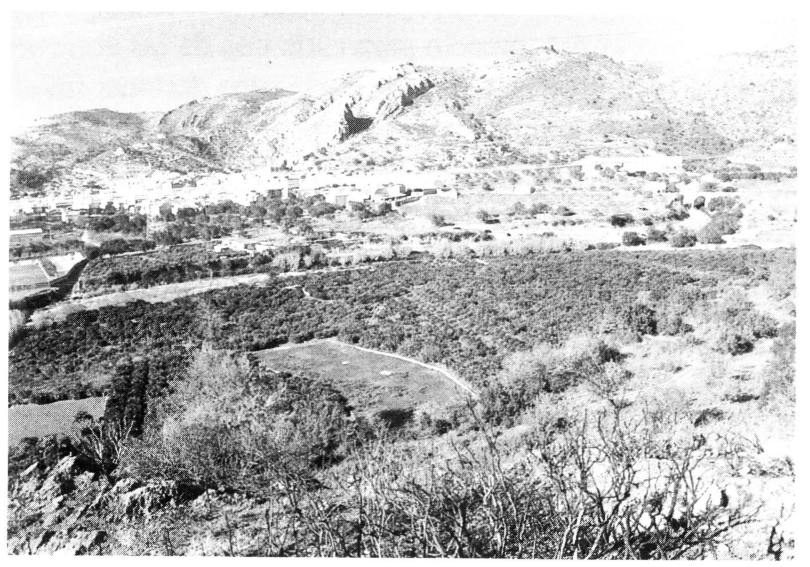

Emplazamiento de la futura variante. Vega del río Borriol.

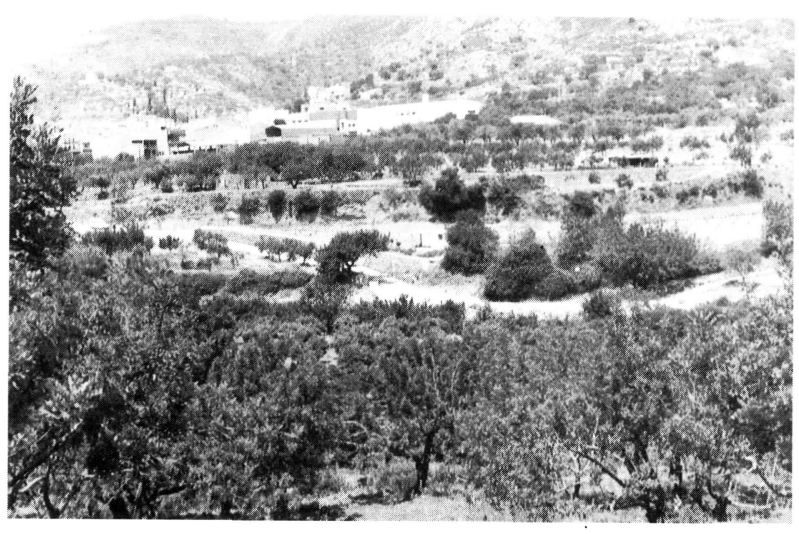

Borriol, casco urbano; al fondo, los cerros que impiden cualquier alternativa al norte.

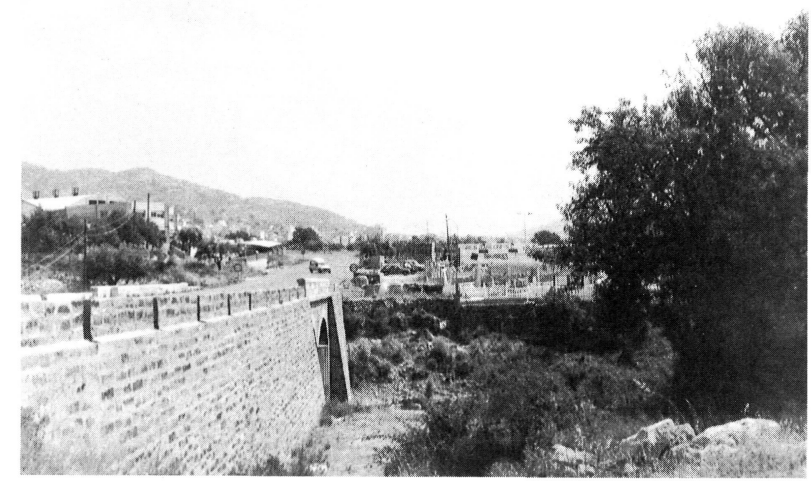

Inicio de la variante. Cauce del río Borriol. 
42

Informes de la Construcción, Vol. 47 ns $441-442$, enero/febrero - marzo/abril 1996

Por otra parte se caracterizaban las acciones que se iban a producir en las fases de construcción y explotación de la carretera, para, de este modo, poder establecer los efectos de estas acciones en el medio, esto es, los impactos.

Una vezidentificados los impactos es preciso cuantificarlos para poderlos medir y poder sumar, de algún modo que resulte significativo, los unos con los otros. Por lo tanto, se procedió a determinar cuáles eran los indicadores de impacto, pues de ese modo se puede determinar la magnitud e importancia del impacto para cada una de las acciones que les iban a afectar. Como ejemplo cabe destacar los siguientes indicadores de impacto:

Geologia: procesos erosivos, inestabilidad de laderas, deslizamientos,

Hidrología: afecciones al flujo subterráneo, calidad de las aguas, régimen hidráulico,.

Comunidades biológicas: pérdida de especies, pérdidas de cultivos, ...

Atmósfera: variaciones de las condiciones microclimáticas, contaminación atmosférica, contaminación acústica,...

Paisaje: alteración del paisaje
Socioeconomía: efecto barrera, variación de la actividad industrial,...

Se empleó el Método de la Matriz de Leopold para llevar a cabo la evaluación de impacto para las alternativas. Ninguna de ellas dió lugar a un nivel de impacto crítico, siendo las más favorables las alternativas que discurrían por la vega del río Borriol (las 3 y 4 del plano), cuyo nivel de impacto se estimó como impacto moderado.

En el Anejo "Elección de la solución definitiva. Análisis Multicriterio" de la Memoria del Proyecto Básico se emplearon las conclusiones del Estudio de Impacto Ambiental, extraídas hasta este punto. La elaboración del Estudio de Impacto Ambiental fue en todo momento simultaneada con la redacción del Proyecto Básico, coordinado por el equipo técnico de INTRAESA; de este modo, las conclusiones de las primeras fases del Estudio de Impacto Ambiental fueron introducidas en el Análisis Multicriterio del Proyecto Básico, y cuando de este proceso resultó seleccionada una alternativa, se desarrollaron las fases siguientes del Estudio de Impacto Ambiental para la misma.

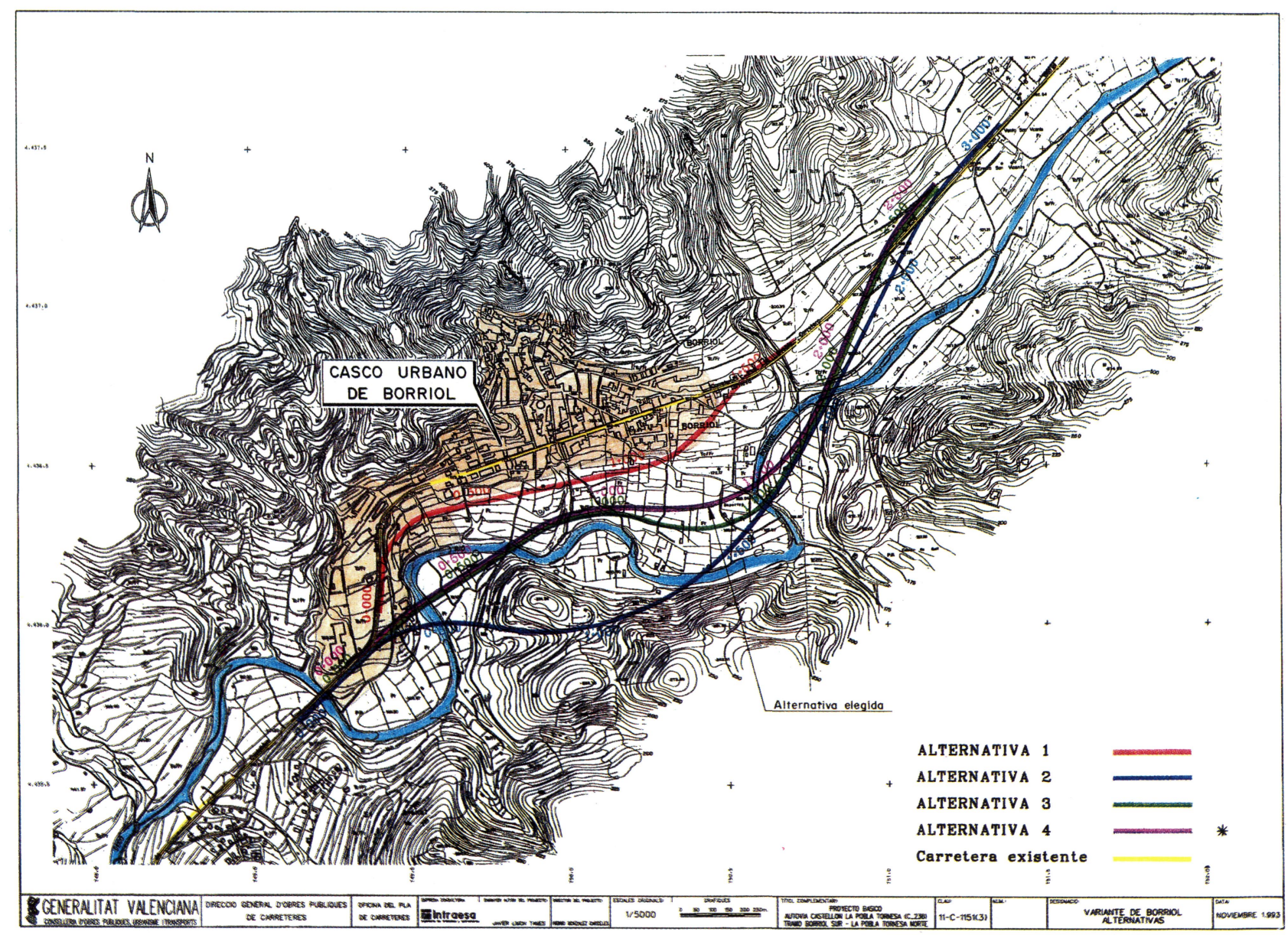

Plano de Alternativas. 
De las dos alternativas consideradas como de menor impacto, el análisis multicriterio (se empleó una técnica blanda, en concreto el método "Electre I") concluyó que la más adecuada era la alternativa que, discurriendo por la vega del ría Borriol, tenía un trazado más favorable al ser los radios de las alineaciones curvas mayores (Alternativas 4). Debe tenerse en cuenta que se estaba estudiando como autovía y, por tanto, la velocidad específica interesaba que fuese la mayor dentro de las posibles.

Como última fase del Estudio de Impacto Ambiental, se definieron las medidas correctoras de impacto que, a juicio de los técnicos de INTRAESA y en vista del tipo de impactos que previsiblemente se producirían como consecuencia de las obras, en sus fases de construcción y explotación, eran las más adecuadas. Se marcaron los objetivos que se perseguían con ellas, como el restablecimiento de la cubierta vegetal, la no alteración del cauce del río Borriol, etc.

Algunas de las medidas correctoras de impacto propuestas:

Fase de Construcción:

-Ejecución de los taludes atendiendo a consideraciones geotécnicas y paisajísticas simultáneamente.

-Reposición de servicios afectados, tanto de Compañías, como caminos y accesos a todas las parcelas que en la actualidad los tienen.

-Estudio detallado de yacimientos y canteras para los materiales necesarios en la construcción de las obras, así como elección cuidadosa de los vertederos para los sobrantes de las excavaciones.

-Recuperación de tierra vegetal y posterior empleo en las bermas.

-Plantación y revegetación de taludes con aliaga (Genis- ta Scorpius) y estepa (Cistus Laurifolius)

-Plantación de las medianas con adelfas (NeriumAleander) y pitosporos (Pitosporum Tobirá)

-Plantación de árboles en los grandes espacios expropiados entre los ramales de los enlaces; en concreto se proponían pinos (Pinus Halepensis, Pinea y Nigra) y encinas (Quercus Ilex).

-Protección de los terraplenes con escollera en las zonas consideradas como inundables por el río Borriol

-Construcción de una pista ciclista desde Borriol hasta La Pobla Tornesa para evitar la circulación de bicicletas por la futura autovía, pero manteniendo la posibilidad de seguir empleando este medio de transporte.

Como Documento Anejo al Estudio de Impacto Ambiental se elaboró un Estudio Arqueológico exhaustivo de la zona de emplazamiento de las obras para determinar la posible existencia de yacimientos que fuese necesario mantener in situ por cuestiones legales, lo que hubiese obligado a desechar aquel trazado necesariamente. Aunque indicó la existencia de muchos restos arqueológicos interesantes, los expertos determinaron que sería suficiente con la recogida y catalogación de los restos hallados a lo largo de toda la fase de movimiento de tierras de la obra.

Asimismo, ya petición de la Ofícina del Plá de la C.O.P.U.T., se hizo un estudio en profundidad del impacto acústico en cada una de las viviendas y edificaciones que quedaban a una distancia inferior o igual a 100 metros de la alternativa seleccionada, para determinar la sensación sonora -y el incremento de la misma en los tramos de duplicación de la calzada existente- $y$, de esta forma proyectar y valorar la necesidad de barreras antirruido allí donde la sensación sonora, o el incremento de la misma, resultasen intolerables $(S>70 \mathrm{~dB}$ o $\mathrm{S}>10 \mathrm{~dB})$. Las conclusiones de dicho estudio fueron que en ningún caso la sensación sonora era intolerable en la variante de Borriol.

\begin{tabular}{||c|c|c|c|c|c|c||}
\hline$N^{\circ}$ & D.O. & MARGEN & TIPO EDIFICIO & $\begin{array}{c}\text { DISTANCIA } \\
\text { AUTOVIA }\end{array}$ & $\begin{array}{c}\text { SENSACIÓN } \\
\text { SONORA (dB) }\end{array}$ & PERCEPCIÓN \\
\hline 1 & $0+040$ & IZQDA & Fábrica cerámica & $20 \mathrm{~m}$ & 62,28 & TOLERABLE \\
\hline 2 & $0+060$ & DCHA & Fábrica cerámica & $18 \mathrm{~m}$ & 67,38 & TOLERABLE \\
\hline 3 & $0+320$ & IZQDA & Casa & $12 \mathrm{~m}$ & 65,67 & TOLERABLE \\
\hline 4 & $0+960$ & IZQDA & Casa & $55 \mathrm{~m}$ & 57,67 & ACEPTABLE \\
\hline 5 & $1+220$ & IZQDA & Casa & $48 \mathrm{~m}$ & 58,86 & ACEPTABLE \\
\hline 6 & $1+240$ & DCHA & Casa & $65 \mathrm{~m}$ & 56,22 & ACEPTABLE \\
\hline 7 & $1+380$ & DCHA & Casa & $25 \mathrm{~m}$ & 64,52 & TOLERABLE \\
\hline 8 & $1+520$ & IZQDA & Instalac. deportivas & $73 \mathrm{~m}$ & 55,38 & ACEPTABLE \\
\hline
\end{tabular}




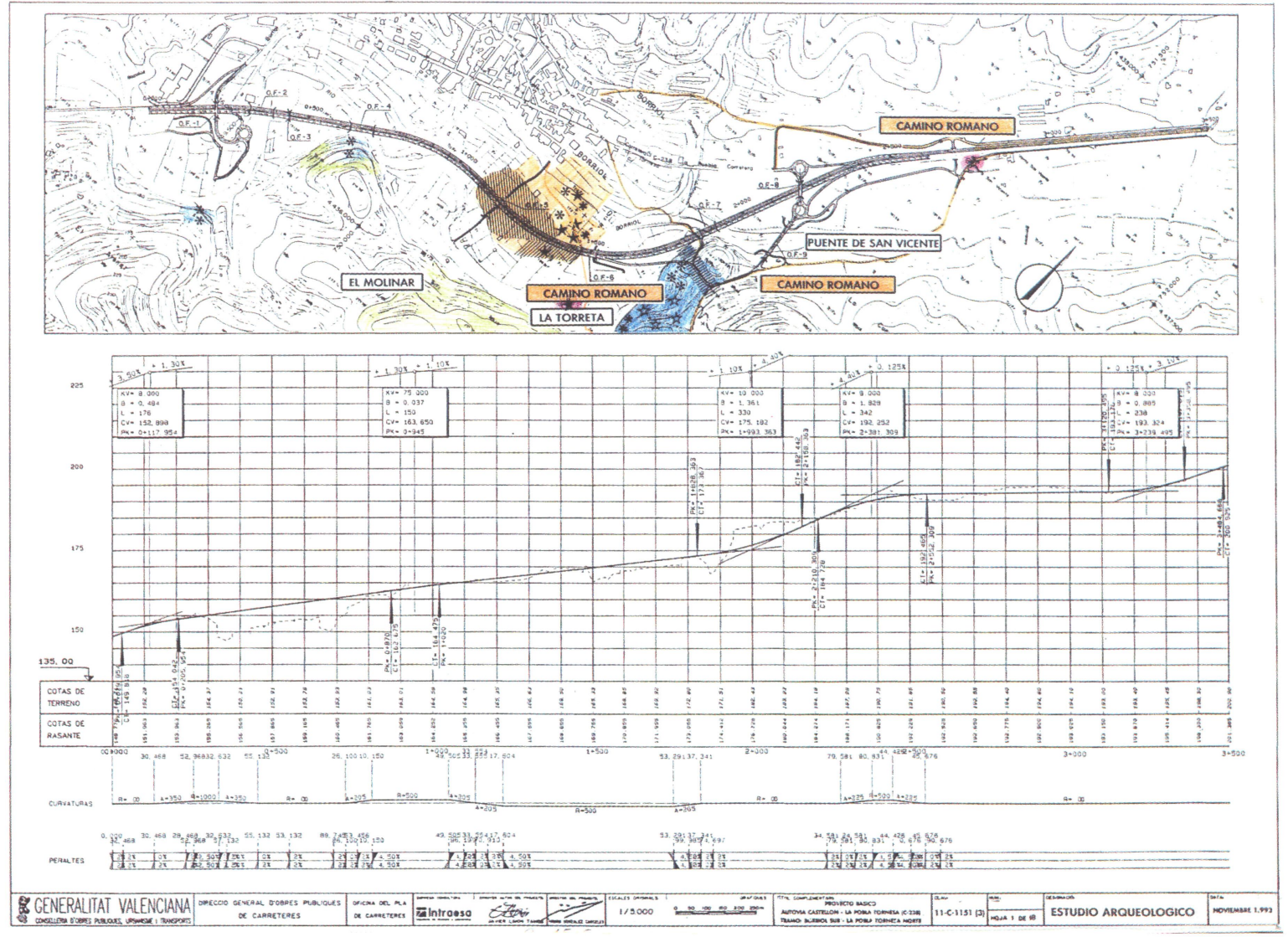

Estudio arqueológico.

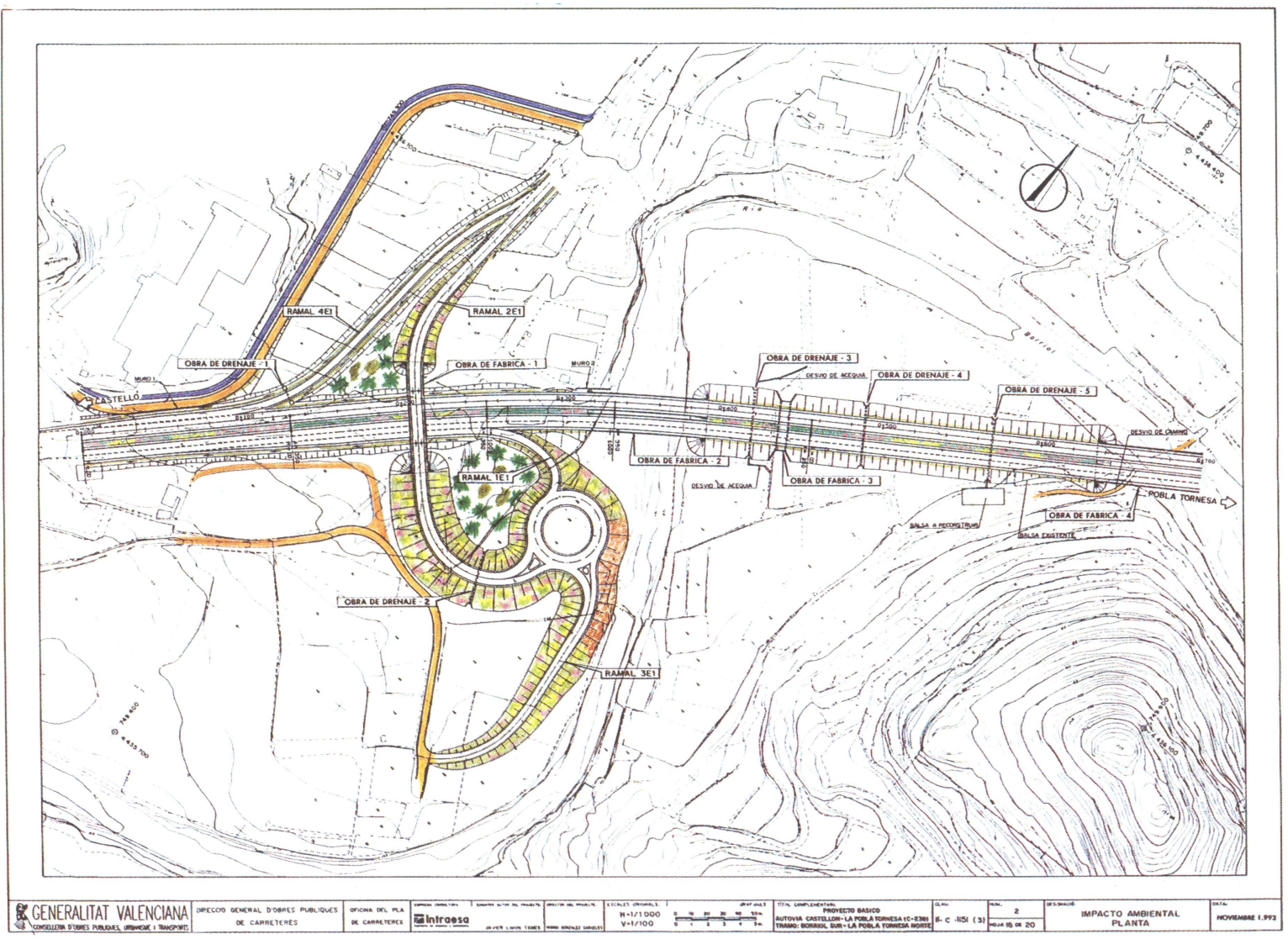




\section{Información pública y declaración de impacto}

En el mes de julio de 1994, el Proyecto Básico y de Expropiaciones "Autovía Castellón - La Pobla Tornesa (C-238). Tramo: Borriol Sur-La Pobla Tornesa Norte" fue expuesto a información pública en el Ayuntamiento de la localidad de Borriol. Se informó de esta circunstancia a todos los organismos que pudieran verse afectados por las obras.

Se recogieron algunas alegaciones de habitantes de la localidad, propietarios de parcelas afectadas por las expropiaciones. Todas ellas hacían referencia a las superficies de expropiación o a confusiones en los datos de los propietarios. Ninguna implicaba modificación alguna en el Proyecto.

La Confederación Hidrográfica del Júcar, a cuya cuenca pertenece el río Borriol, envió a la C.O.P.U.T. un escrito por el que manifestaba su acuerdo con los cálculos hidrológicos y de drenaje que se habían llevado a cabo por INTRAESA en al Proyecto Básico, aceptando el dimensionamiento de todas las obras de drenaje previstas.

El 21 de octubre de 1994, la Direcció General de Qualitat Ambiental de la Consellería de Medi Ambient de la Generalitat Valenciana formuló la Declaración de Impacto Ambiental sobre el Proyecto Básico y de Expropiaciones. En ella se decía:

(......) Por todo ello y en uso de las facultades que tengo legalmente atribuidas, formulo la siguiente

\section{DECLARACION DE IMPACTO AMBIENTAL}

\section{Primero}

Estimar aceptable, a los solos efectos ambientales, la realización de las obras del Proyecto "Autovia CastellónLa Pobla Tornesa (C-238). Tramo: Borriol Sur-La Pobla Tornesa Norte", clave 11-CS-1151(3), de acuerdo con las previsiones del Estudio de Impacto Ambiental y de las condiciones y precisiones siguientes:

1) Debido a la posibilidad de afectar a yacimientos arqueológicos, puestos de manifiesto en el Estudio de Impacto Ambiental, si durante la construcción de las obrasse encontraran restos arqueológicos, se comunicarán los hallazgos a la Dirección General de Patrimonio Artístico, de la Consellería de Cultura, de acuerdo con la Ley 16/1985 de Patrimonio Histórico Español.

2) Los materiales de préstamo (......) provendrán de canteras o lugares de extracción ambientalmente correctos y convenientemente legalizados, (......)

3) Si fuera necesario utilizar un vertedero (......) se deberá someter al procedimiento de Evaluación de Impacto 1 mbiental.
4) En el Proyecto Definitivo se contemplarán y resolverán las afecciones a las diversas vias pecuarias existentes en la zona, que a continuación se indican: (......)

5) A pesar que en el Estudio de Impacto Ambiental se contempla un Estudio de Ruido en el que se concluye que la sensación sonora media es aceptable, si durante la fase de explotación de la carretera los niveles de inmisión sonora medidos (......) sobrepasaran los $55 \mathrm{~dB}$ durante la noche (......) y los $65 \mathrm{~dB}$ durante el dia (......), se diseñarán y ejecutarán las medidas necesarias para reducirlas.

6) (......) se velará para que (......) se proceda a la retirada de todos aquellos escombros, materiales sobrantes, (...

..) así como a la adecuación y limpieza (......).

7) No se almacenará ni ubicará ningún tipo de elemento (......) en los diferentes tramos en que es atravesado el cauce del rio Borriol por la carretera, y que pueda verse afectado por avenidas. Asimismo queda prohibido cualquier tipo de vertido (.......).

8) Los taludes, (......) se revegetarán extendiendo una capa de tierra vegetal sobre su superficie y una posterior siembra con especies subarbustivas autóctonas. (.......)

9) Referente al Plan de Revegetación propuesto en el Estudio de Impacto Ambiental, se sustituirán las especies "aliaga" (Genista Scorpius) y "estepa" (Cistus Laurifolius) por otras autóctonas de la zona, entre las que se sugieren lassiguientes: "CoronillaJuncea", "Cistus Albidus", "Lavandula Dentada", "Lonicera implexa", etc...

$(\ldots / \ldots)$

Como se aprecia, la Declaración de Impacto vino a subrayar lo indicado en el Estudio de Impacto Ambiental del Proyecto, haciendo hincapié en aquellas cuestiones que, por la experiencia en casos similares, resultan prioritarios para esta Administración Autonómica.

\section{Proyecto de construcción: materialización de las medidas correctoras}

Una vez formulada la Declaración de Impacto y habiendo sido aprobado administrativamente el Proyecto Básico y de Expropiaciones, se adjudicó a la misma Empresa Consultora INTRAESA la redacción del Proyecto de Construcción "Variante de Borriol de la C-238". De acuerdo con la Orden de Estudio, debían proyectarse las obras de la variante en carretera, es decir, con una sola calzada, a la localidad de Borriol, de acuerdo con el trazado desarrollado en el Proyecto Básico.

El proyecto comprende, por tanto, los primeros 2.870 
metros del total del Proyecto Básico. La calzada que se proyecta es la izquierda de la futura autovía.

En este Proyecto se respetan las estructuras proyectadas para los enlaces en el Proyecto Básico, aptas para el paso de las dos calzadas bajo ellas. El resto de estructuras se diseñan para la carretera y deberán ensancharse -caso de los marcos- o duplicarse -caso de los puentes sobre el río Borriol- cuando se haga la autovía.

El Proyecto de Construcción consta de los cuatro Documentos habituales; un Anejoa la Memoria(Documento $\mathrm{N}^{\circ} 1$ ) es "Medidas Correctoras de Impacto Ambiental". En él se recogen todas las medidas previstas en el Estudio de Impacto Ambiental, así como las modificaciones y ampliaciones a que hace referencia la Declaración de Impacto. Otro Anejo, "Yacimientos y Canteras", recoge toda la información existente sobre yacimientos y canteras en la zona de emplazamiento de las obras, para dar cumplimiento a las indicaciones de la Declaración de Impacto.

En el Documento $\mathrm{N}^{\circ} 2$, Planos, se reflejan: los taludes de desmonte y terraplén a ejecutar; las plantaciones y su disposición; la señalización de las vías Pecuarias para su perfecta identificación. En el Documento $N^{0} 3$, Pliego de Prescripciones Técnicas Particulares, se definen las cualidades de todas las plantaciones a emplear, así como los métodos de plantación y posterior cuidado no sólo hasta la Recepción Provisional de las Obras, sino durante todo el período de garantia.

En el Documento $\mathrm{N}^{\circ} 4$, Presupuesto, están contempladas las plantaciones a utilizar, la tierra vegetal, los carteles (fuera de normativa de Carreteras) de señalización de las vías pecuarias, y se prevén partidas alzadas para las prospecciones arqueológicas, para los trabajos de catalogación de los hallazgos y para la elaboración de los informes pertinentes.

El Proyecto de Construcción "Variante de Borriol de la C-238" ha sido aprobado por las autoridades competentes de la C.O.P.U.T. de la Generalitat Valenciana en fechas recientes.

Cuando se liciten las obras objeto del mismo, el proceso de Evaluación de Impacto continuará, en las fases de construcción y de explotación de la carretera. Deberá redactarse un Programa de Vigilancia Ambiental, basado en el propuesto en el Estudio de Impacto Ambiental del Proyecto Básico de referencia. Se procederá a realizar las medidas de ruido que indica la Declaración de Impacto, para confirmar las previsiones hechas y, en caso contrario, proyectar y construir las pantallas antirruido donde fuesen necesarias.

En definitiva, la primera etapa de la Evaluación de Impacto Ambiental para la variante de la carretera C-238 a su paso por la localidad de Borriol (Castellón) está terminada. Resta la parte práctica en la que se debe materializar lo proyectado hasta ahora.

Deseo hacer énfasis en la importancia de la parte ya cubierta del proceso, la fase de Proyecto, pues de su precisión o deficiencias dependerá el resultado final. Las operaciones que se lleven a cabo se limitarán, en su mayor parte, a cumplir lo proyectado y sólo se rectificarán las actuaciones en caso de demostrar no ser las adecuadas, lo cual provocaría un gran retraso en la recuperación ambiental y un incremento en los costes. 